\title{
DEVELOPMENT AND APPLICATIONS OF A 3D COMPRESSIBLE NAVIER- STOKES SOLVER
}

\author{
H. Kuerten, B. Geurts, J. van der Burg, B. Vreman, P. Zandbergen
}

Department of Applied Mathematics, University of Twente, P.O. Box 217, 7500 AE Enschede, The Netherlands

\section{Introduction}

As a part of the Dutch ISNaS project our group and NLR jointly develop a flow solver for compressible, turbulent flow. This flow solver is especially aimed at applications on the industrial level: the multi-element airfoil and wing/body combination, both at transonic flow conditions. The flow solver is based on the Reynolds-averaged Navier-Stokes equations, in which presently the algebraic Baldwin-Lomax turbulence model is adopted. In reference [1] the first results, for laminar and turbulent flow around a single airfoil and over a finite flat plate have been shown. In the present paper recent developments in the solver are discussed.

In section 2 the numerical method used in the ISNaS solver is briefly described. Section 3 discusses the role of the numerical, or artificial dissipation in relation to the physical dissipation. In section 4 numerical aspects of the extension of the monoblock solver to a multiblock solver are described. The numerical method used in the ISNaS solver serves as a basis for many CFD programs used in our group. These programs are not only intended for the two applications mentioned above, but also for more fundamental studies of turbulence (with the help of large eddy simulation (LES) and direct numerical simulation (DNS)) and for the simulation of viscous water waves. In section 5 of this paper the use of the numerical method in large eddy simulation is discussed.

\section{Numerical Method}

The formulation of the numerical method starts from the integral formulation of the unsteady Reynolds-averaged Navier-Stokes equations, in which the densities of the conserved quantities are used as dependent variables. This integral formulation leads in a natural way to a finite volume approach in physical space, preserving the conservation property. A cell-vertex method with overlapping control volumes is used, since it remains accurate in the neighbourhood of grid distortions, which are unavoidable in the relevant applications. In figure 1 the control volumes used for the convective and viscous fluxes are sketched.

For the spatial discretization central differencing is applied with additional second-and fourth order nonlinear artificial dissipation. The formulation of the artificial dissipation is based on the work of Jameson [2], and is described in more detail in the next section. At a solid surface the velocity components are set equal to zero, while the density and energy density are obtained by solving their conservation equations, assuming an adiabatic wall. 

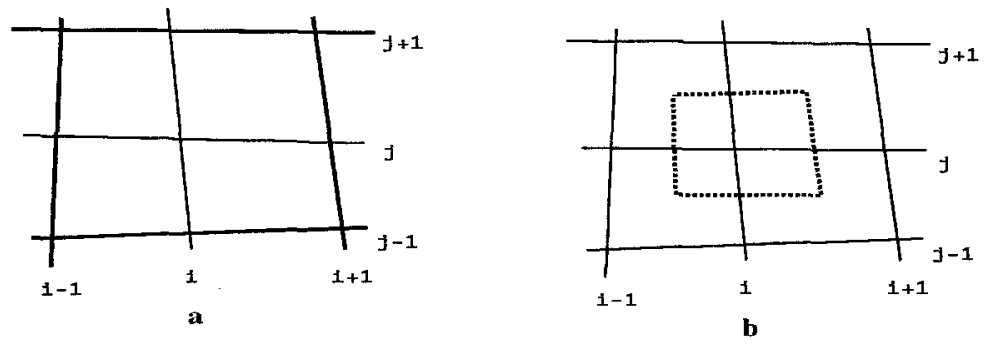

Figure 1: Control volumes used for the convective terms (a) and viscous terms (b)

The treatment of the far-field boundaries is based on approximate Riemann invariants, with the optional inclusion of a circulation correction [3].

The system of ordinary differential equations which results from the spatial discretization is integrated in time with a time-explicit multistage Runge-Kutta method, where local time stepping is used. The calculation time can drastically be reduced by the use of a multigrid technique and implicit residual averaging. Both for two-dimensional turbulent flow around an airfoil and three-dimensional turbulent flow around a wing the gain in calculation time, using these acceleration techniques is of the order of a factor of 10 .

For calculations on smooth grids instead of the cell-vertex method, sketched in figure 1 , a vertex-based method can be used as an alternative. This method differs in the calculation of the convective fluxes, for which the same control volume is used as for the viscous fluxes. This and the fact that the control volume on which the artificial dissipation method is based is the same, increase the consistency of the method.

\section{Artificial Dissipation}

In refs. [1], [4] it has been shown that the numerical method described in the previous section captures the physics accurately for subsonic, laminar flow over a flat plate. For transonic, turbulent flow at high Reynolds number, however, two disadvantages of the usual formulation of artificial dissipation [2], [5] become apparent. In the first place, shock waves are not accurately captured, and in the second place, the artificial dissipation in the boundary layer is rather high, leading to too high drag coefficients. In this section it is shown that these drawbacks can be met by several modifications in the formulation.

After spatial discretization the Navier-Stokes equations can schematically be written as $d w / d t=f_{c}+f_{v}+f_{a}$, where $w$ is the vector of dependent variables in all grid points, $f_{c}$ is the convective flux, $f_{v}$ the viscous flux and $f_{a}$ the artificial dissipation. The latter consists of contributions from every spatial direction, each of which has the form $d_{i+1 / 2}-d_{i-1 / 2}$, where

$$
d_{i+1 / 2}=S_{i+1 / 2}\left[\epsilon_{i+1 / 2}^{(2)} \Delta_{i+1 / 2} w-\epsilon_{i+1 / 2}^{(4)} \Delta_{i+1 / 2}^{3} w\right]
$$

Here, $S_{i+1 / 2}$ is a scaling factor, $\epsilon_{i+1 / 2}^{(2)}$ and $\epsilon_{i+1 / 2}^{(4)}$ are functions of a shock sensor, and $\Delta_{i+1 / 2}$ and $\Delta_{i+1 / 2}^{3}$ are first and third order difference operators.

An easy way to reduce the artificial dissipation in the boundary layer, where the viscous dissipation should be dominant, is a multiplication of $S_{i+1 / 2}$ with a function of the local Mach number. Application of this scaling in the direction normal to a solid wall with a linear function leads to a significant reduction of the drag coefficient.

For two-dimensional calculations on meshes with grid cells of high aspect ratio the scaling factor $S_{i+1 / 2}$ in the $i$-direction is usually taken as $S_{i}=\left[1+\left(\lambda_{j} / \lambda_{i}\right)^{2 / 3}\right] \lambda_{i}$, where $\lambda_{i}$ is the 
maximum eigenvalue of the flux Jacobian matrix in the $i$-direction [5]. The term between the brackets increases the artificial dissipation in the streamwise direction in the boundary layer. It appears that in the vertex-based discretization $S_{i}=\lambda_{i}$ can be chosen, at the cost of a small reduction in multigrid efficiency. However, the decrease of artificial dissipation leads to a better caption of the physical phenomena in the solution, which would otherwise require a finer grid.

Shock waves can be captured more accurately, if the first order difference terms in the artificial dissipation, which are triggered by a shock sensor, are replaced by upwind differences [6]. This approach works both for inviscid and turbulent, viscous flow problems (see figure 2).

\section{Numerical Aspects of Multiblock Solver}

For the multi-element airfoil application a multiblock solver will be constructed. The total computational domain will be divided into blocks in such a way that in each block a structured, boundary-conforming grid can be adopted. This block structure opens the possibility to solve the Euler equations in those blocks which are situated in the 'outer' regions of the flow, and hence save calculation time. The numerical aspects of the multiblock solver have been investigated, using a multiblock structure around a single airfoil [7].

To this end each of the blocks is taken out of the total domain separately and 'dressed' with two rows of dummy grid points. The blocks can be updated over one or more time steps independently, whilst keeping the variables in the dummy grid points frozen. After each block has been treated in this way the variables at the block interfaces are averaged.

It appears that the steady-state solution and convergence behaviour are unchanged, irrespective of the number of blocks and location of the interfaces, if the number of time steps over which the dummy variables are kept frozen is not too large [7]. Further, the solution is unchanged, if outside the boundary layer the Euler equations are solved instead of the Navier-Stokes equations (see figure 3), even if the grid is distorted near the interface.

\section{$5 \quad$ Large Eddy Simulation}

Especially for complex flows, with large separation regions and shock-boundary layer interaction, the results of flow simulation based on the Reynolds-averaged Navier-Stokes equations inadequately describe related physical experiments. This is mainly caused by the turbulence model. Information on improvements of turbulence models can be obtained through comparison with results from large eddy simulations of flow in simpler geometries. It has been shown that large eddy simulations of compressible flow are possible within a finite-volume approach, if the discretizations of the convective and viscous fluxes are performed on the same control volume [8]. If the Simpson rule is used for the integration of both fluxes, the theoretically expected velocity correlation spectrum is obtained in the case of homogeneous, isotropic, decaying turbulence in a 3D box. With the cell-vertex method direct numerical simulations of turbulent flow in a compressible mixing layer have been performed. The pairing of vortices and the correlation between pressure and vorticity are in agreement with literature.

\section{Acknowledgements}

The authors are greatly indebted to D. Dijkstra and F.J. Brandsma for many stimulating discussions. This work is partly subsidized by the Dutch Ministries of Education and Sciences and of Transport and Public. Works. Part of the necessary supercomputer time was 


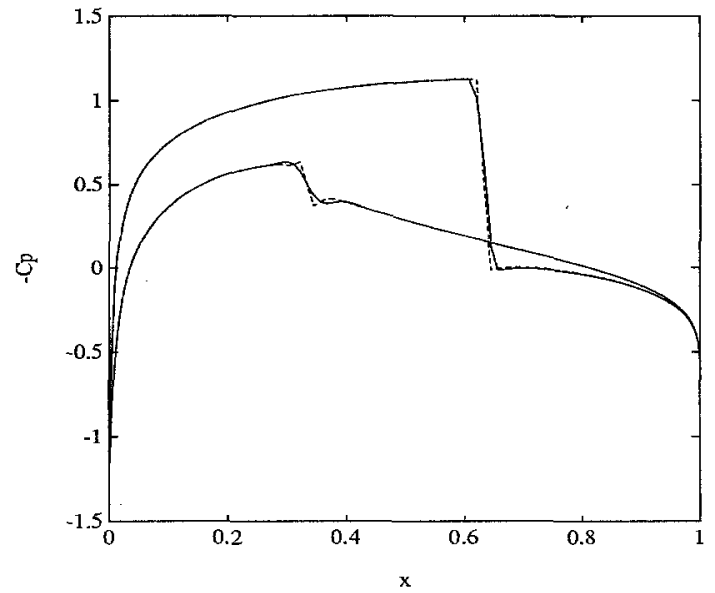

Figure 2: Pressure coefficient on the airfoil for inviscid flow around a NACA0012 profile at $M_{\infty}=0.8$ and $\alpha=1.25^{\circ}$; central differences in artificial dissipation (solid) and upwind differences (dashed).

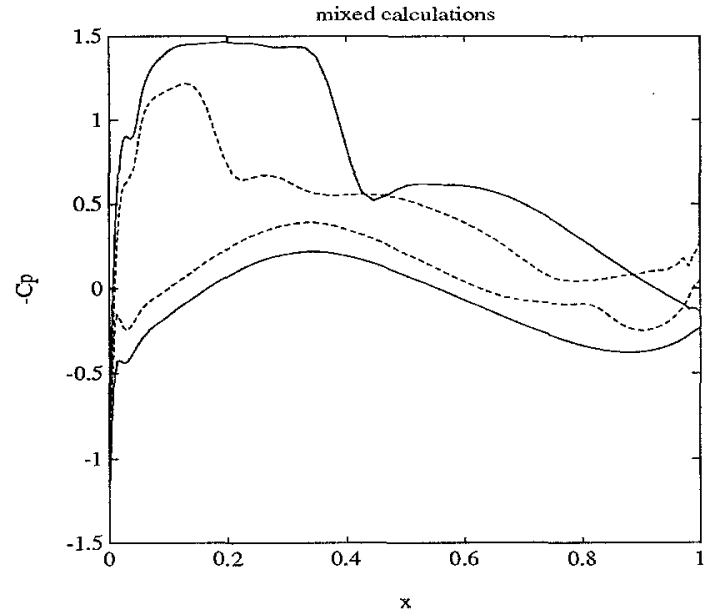

Figure 3: Pressure coefficient on the airfoil for turbulent flow around a CAST7 profile at $M_{\infty}=0.7, R e=4 \times 10^{6}$ and $\alpha=2^{\circ}$; only Navier-Stokes (solid), Euler-Navier-Stokes interface just outside boundary layer (dotted, coincides with solid) and just inside boundary layer (dashed). 
sponsored by the National Computing Facilities Foundation, NCF, with financial support from the Netherlands Organisation for Scientific Research, N.W.O.

\section{References}

[1] F.J. Brandsma, J.G.M. Kuerten: The ISNaS compressible Navier-Stokes solver; first results for single airfoils, in: K.W. Morton (Ed.), Tweifth International Conference on Numerical Methods in Fluid Dynamics, Oxford, U.K., July 9-13, 1990, Springer Verlag, pp. 152-156.

[2] A. Jameson, W. Schmidt, E. Turkel: Numerical Solutions of the Euler Equations by Finite Volume Methods Using Runge-Kutta Time-Stepping-Schemes, AIAA Paper 811259 (1981).

[3] J.G.M. Kuerten: Numerical Definition Document for the Time-explicit ISNaS Solver, ISNaS 88.10.031 (1988).

[4] D. Dijkstra, J.G.M. Kuerten: An easy test-case for a Navier-Stokes solver, in: Proceedings of the First European Computational Fluid Dynamics Conference, Brussels, Belgium, September 7-11, 1992.

[5] V.N. Vatsa, B.W. Wedan: Development of an efficient multigrid code for 3-d NavierStokes equations, AIAA Paper 89-1791 (1989).

[6] J.W. van der Burg, J.G.M. Kuerten, P.J. Zandbergen: Improved shock capturing of Jameson's scheme for the Euler equations, to appear in Int. J. Num. Meth. Fluids.

[7] B.J. Geurts, J.G.M. Kuerten: Numerical aspects of a block structured compressible flow solver, to appear in J. Engg. Math.

[8] A.W. Vreman, B.J. Geurts, J.G.M. Kuerten, P.J. Zandbergen: A finite volume approach to large eddy simulation of compressible, homogeneous, isotropic, decaying turbulence, to appear in Int. J. Num. Meth. Fluids. 